\title{
Trellis Coded Partial Response Signaling for Power and Bandwidth Efficient Digital Transmission
}

\author{
Chen-Kun Wang Lin-Shan Lee Yumin Lee \\ Department of Electrical Engineering \\ National Taiwan University \\ Taipei, Taiwan 10764, R.O.C.
}

\begin{abstract}
In this paper we presented a particular approach to design power and bandwidth efficient trellis coded partial response signaling (TCPRS) . Two theorems enable us to find good TCPRS codes based on good codes for trellis coded modulation (TCM). A class of good TCPRS codes is thus found. The free distance and asymptotic coding gain is calculated, and a maximum coding of $4 \mathrm{~dB}$ can be achieved by this class of codes. We also discuss the distance structure of this class of codes, and finally the performance at median signal to noise ratio (SNR) is presented.
\end{abstract}

\section{Introduction}

In most transmission channels, bandwidth is at a premium, hence an important attribute of any attractive digital signaling schemes is its ability to make efficient use of the bandwidth. Many bandwidth efficient signaling schemes bave been devised for both linear and nonlinear channels in the past $[1,2,3]$. One of the most important bandwidth efficient signaling scheme for linear channels is the so-called correlative level coding, or partial-response signaling (PRS), in which a considerable amout of bandwidth can be saved as compared to pulse amplitude modulation (PAM) with very little sensitivity degradation [4]. On the other hand, another important issue in digital transmissions is the error control coding, which provides the capability to combat with the channel impairments. Conventional error control coding strategies achieved coding gains at the expenses of increased bandwidth for binary modulation per dimension, which is undesirable in terms of bandwidth efficiency. This problem was then solved by trellis-coded modulation (TCM) first proposed by Ungerbeock [5], which achieved a coding gain of 3 to $6 \mathrm{~dB}$ without any bandwidth expansion as compared to conventional PAM or quadrature amplitude modulation (QAM). However, since the spectrum of TCM is basically the same as that of conventional PAM or QAM, it still re- quires an excess bandwidth of 15 to 100 percent as compared to the Nyquist bandwidth, which can be achieved by the minimum bandwidth PRS [2]. It's thus natural to try to combine the merits of PRS and TCM altogether, i.e., to achieve an attractive coding gain with the signal spectrum confined within the Nyquist bandwidth. Recently, very much attention has been paid to this direction. Wolf et al. [6] and Calderbank et al. [7] designed trellis codes over binary partial response channels by introducing redundancy in time, where Ketchum [8] and Forney et al. [9] considered a quite different approach of trellis coding for multi-level PRS with expanded signal sets. The coding scheme considered here in this paper is basically the one proposed by Ketchum [8], and will be refered to as trellis-coded partial response signaling (TCPRS) hereafter.

The earlier reports in the literature concluded that exhaustive search for good codes for TCPRS is necessary, and was partially done by Ketchum [ 8 ] by assuming the all-zero code sequence is transmitted. Unfortunately, TCPRS is irregular [10] such that the free distance can not be found this way. In fact, the free distances of the previously reported codes [8] is actually smaller. After the formulation of the code search problem, we provide two powerful theorems at section 4 for precoded TCPRS that reduce the problem of searching for good codes for TCPRS to that of searching for good codes for TCM [5] over memoryless channels. From these two theorems a class of good codes is found. The free distance of these good codes is verified by the generalized transfer function technique [11], and the asymptotic coding gain is found to be limited to $4 \mathrm{~dB}$. We also show in section 4 that the distance structure of TCPRS is dominated by the TCM code used, instead of by the channel memory. Hence the quasi-catastrophic [9] nature of uncoded PRS is not inherited by TCPRS. In section 5 the coding gain at moderate signal to noise ratio is discussed, and finally the conclusion is given in section 5 .

\section{Free distance of uncoded PRS}

The model of a PRS can be characterized by its transfer function $F(D)=\sum_{i=0}^{N-1} f_{i} D^{i}$, where $f_{i}, i=0,1, \cdots, N-1$, are integers. Let the input sequence be represented by

907.6.1 
$x(D)=\sum_{n=0}^{\infty} x_{n} D^{n}$, where $x_{n}$ is an integer between 1 and $M-1$, then the output sequence is $y(D)=\sum_{n=0}^{\infty} y_{n} D^{n}$, where

$$
y(D)=x(D) F(D) .
$$

In some cases, the input sequence is first precoded (if a precoder exists) before it is transmitted through the channel. Then we have the precoded sequence $p(D)$

$$
p(D)=x(D) / F(D) \quad(\bmod M)
$$

and the PRS output

$$
y(D)=p(D) F(D) .
$$

To minimize the error probability of a PRS, maximum likelihood sequence estimation is employed. The asymptotic performance of a PRS at high SNR is determined by the squared free Euclidean distance (ED). It is well known that some PRS schemes, e.g., $1+D^{2 n+1}$ and $1-D^{n}$, have the same ratio of squared free distance over signal energy as memoryless PAM [4], and thus the same asymptotic performance.

In practice the performance of a PRS scheme is degraded by its quasi-catastrophic nature and error propagation in the trellis. A trellis is quasi-catastrophic [9] if the free distance occurs at some error events of infinite duration. The catastrophic nature of a PRS will make the effective free distance smaller than its free distance, since a practical Viterbi decoder processes the received signal in finite delay. The error propagation is a side product of the quasi-catastrophic trellis, since error events of such a trellis can be very long and cause a long string of errors in the decoded output. Precoding technique can be used to eliminate the error propogation $[4,9]$, but the trellis remains quasi-catastrophic.

\section{System model of TCPRS}

The discrete model of a TCPRS system is depicted in Figure 1 . With an input alphabet of size $2^{m}$, the convolutional encoder and signal mapper together generate a TCM signal selected from a $2^{m+1}$-element signal set. The TCM encoder formed by the convolutional encoder and signal mapper is essential the type proposed by Ungerboeck [5], namely a rate $m /(m+1)$ systematic convolutional encoder with feedback and a signal mapper that follows the set partitioning rule. For one dimensional modulation $m$ can be any positive integer. For two dimensional modulations, $m$ has to be odd so that the $2^{m+1}$ signal points form the shape of a square to fit into a QPRS channel. If the PRS is not precoded, the TCM signal goes directly to the PRS channel. If the PRS is precoded, the TCM signal is first fed into a precoder, and then to the PRS channel.

The system model and decoding process of the TCPRS system are best described by a finite-state machine (FSM) model [12]. Define the state $\rho_{n}$ of the TCM encoder as the contents of the registers of the systematic encoder at time $n T$, and let the input to the TCM encoder be $b_{n}$ at time

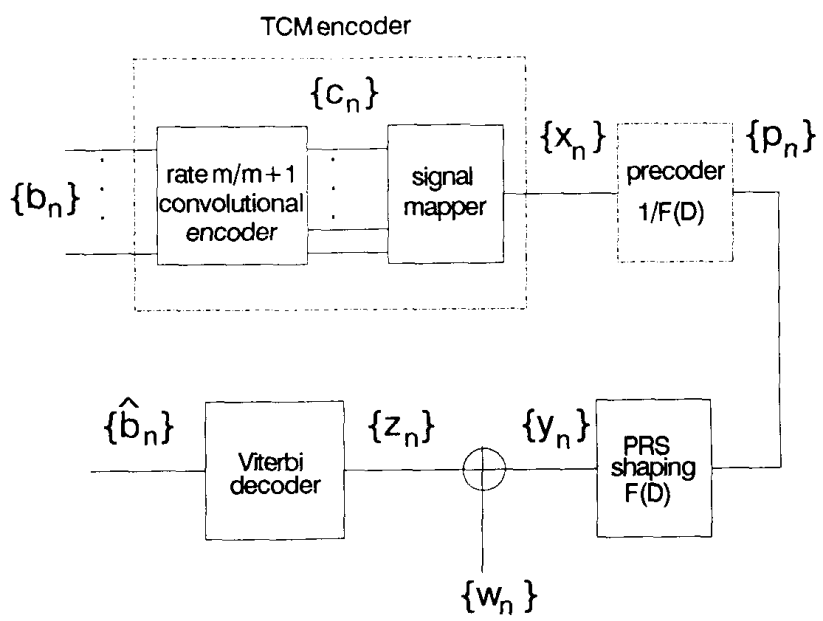

Figure 1. Discrete model of the TCPRS system.

$n T$. The encoded TCM output is then

$$
x_{n}=g_{1}\left(b_{n}, \rho_{n}\right),
$$

and the state transition is

$$
\rho_{n+1}=g_{2}\left(b_{n}, \rho_{n}\right),
$$

where $g_{1}(\cdot)$ is the output function and $g_{2}(\cdot)$ is the state transition function of the TCM encoder. Define the state $\mu_{n}$ of the PRS channel at time $n T$ as the latest $N-1$ inputs, where $N-1$ is the degree of the transfer function $F(D)$, then

$$
\mu_{n}=\left(x_{n-1}, x_{n-2}, \cdots, x_{n-N+1}\right)
$$

if the PRS is not precoded, and

$$
\mu_{n}=\left(p_{n-1}, p_{n-2}, \cdots, p_{n-N+1}\right)
$$

if it is precoded. The state $s_{n}$ of the TCPRS system is then simply the Cartesian product of the state of TCM and that of PRS:

$$
s_{n}=\left(\rho_{n} ; \mu_{n}\right) .
$$

The TCPRS system is now described by the output function $g_{3}(\cdot)$

$$
y_{n}=g_{3}\left(b_{n}, s_{n}\right)
$$

and the state transition function $g_{4}(\cdot)$,

$$
s_{n+1}=g_{4}\left(b_{n}, s_{n}\right) \text {. }
$$

The received signal is the additive-noise-corrupted version of $y_{n}$,

$$
z_{n}=y_{n}+w_{n},
$$

where $y_{n}$ denotes a real or complex discrete channel signal transmitted at time interval $n T$, and $w_{n}$ is a real or complex zero mean Gaussion noise with variance $N_{0} / 2$ per dimension. The SNR is defined as

$$
\mathrm{SNR}=\frac{E\left\{\left|y_{n}\right|^{2}\right\}}{N_{0}}
$$

The receiver is a Viterbi decoder for the FSM described 
above. Performance evaluation is then to determine the coding gain with respect to uncoded PRS or QPRS based on the SNR defined above.

\section{Code search problem for TCPRS}

The problem we want to solve is to specify the parity check polynomials $\boldsymbol{H}(D)=\left[H^{0}(D), H^{1}(D), \cdots, H^{m}(D)\right]$ for the TCM encoder such that the squared free Euclidean distance (ED):

$$
d_{f r e e, T C P R S}^{2}=\min _{y(D) \neq y^{\prime}(D)}\left[\sum_{n} d^{2}\left(y_{n}, y_{n}^{\prime}\right)\right]
$$

is as large as possible. For precoded TCPRS, we have the following relationship from Equation 2 and 3:

$$
y(D)=x(D) \bmod M
$$

where $M$ is $2^{m+1}$ for one-dimensional TCPRS or $2^{(m+1) / 2}$ for two-dimensional TCPRS. The congruent relation between $x(D)$ and $y(D)$ assures that a set partitioning for $x(D)$ is also a set partitioning for $y(D)$. And thus we are able to prove the relationship between the squared free distance of TCM and its corresponding TCPRS:

Theorem 1 The squared free distance of a precoded TCPRS is larger or equal to the squared free distance of the constituent TCM.

For $1 \pm D$ PRS we can prove even tighter bounds:

Theorem 2 For $1 \pm D$ PRS, if the squared free distance of the TCM occurs between two different trellis of multiple $T$ durations, then $d_{\text {free,TCPRS }}^{2} \geq d_{\text {free }, T C M}^{2}+1$ when $d_{\text {free, } T C M}^{2}$ is odd, and $d_{\text {free,TCPRS }}^{2} \geq d_{\text {free,TCM }}^{2}$ when $d_{\text {free, TCM }}^{2}$ is even. If the squared free distance of the TCM occurs only among parallel transitions, $d_{\text {free,TCM }}^{2}$ is even and $d_{\text {free,TCPRS }}^{2} \geq$ $d_{\text {free,TCM }}^{2}+2$.

These two theorem state that a good code for TCM is also a good code for TCPRS. Exhaustive search for good TCM codes has been done [5] with relative ease because the free distance can be found by assuming the all zero sequence is transmitted. We adopt these codes for TCPRS and find the squared free distance. The result is shown in Table 1 for $1 \pm D$ PRS. The readers can see that Theorem 2 is satisfied with equality. For other PRS, we cannot find by computer search any one that Theorem 2 does not hold, but we cannot prove it. The tightness of the bounds in the two theorems suggests that the distance struture of the trellis of TCPRS is dominated by the sub-trellis contributed by the TCM encoder. The length of the error events that contribute to the free distance of TCPRS is only increased by 1 or 2 as compared to the corresponding error event in TCM. Hence the quasi-catastrophic phenomenon associated with uncoded PRS is eliminated in TCPRS.

The asymptotic coding gain of TCPRS is defined as

$$
\text { asymptotic gain }=10 \log \frac{\left(d_{\text {free }}^{2} / S\right)_{\text {coded }}}{\left(d_{\text {free }}^{2} / S\right)_{\text {uncoded }}} \text {, }
$$

where $\mathrm{S}$ is the signal power. Let $S_{x}$ be the signal power of TCM and $S_{y}$ be the signal power of the corresponding TCPRS. We find the relationship:

$$
S_{y}=S_{x} \cdot \sum_{i=0}^{N-1} f_{i}^{2} .
$$

Since the increase of squared free distance of TCPRS over that of TCM is very small, and that $\sum_{i=0}^{N-1} f_{i}^{2} \geq 2$, a loss in coding of at least $3 \mathrm{~dB}$ as compared to TCM is obvious. For $1 \pm D$ PRS, the coding gain is $4 \mathrm{~dB}$ in maximum, two $\mathrm{dB}$ less than TCM can achieve. And if $\sum_{i=0}^{N-1} f_{i}^{2}$ is larger than 2, the gain will further decrease. Hence the PRS that can be used for TCPRS are the one with $\sum_{i=0}^{N-1} f_{i}^{2}=2$. The classes of $1+D^{2 n+1}$ and $1-D^{n}$ PRS are especially recommended, since they posses a spectral null either at dc or at the Nyquist bandwidth.

\begin{tabular}{|r|r|r|r|r|r|r|r|}
\hline$V$ & $\begin{array}{c}\text { encoder } \\
\text { check } \\
\text { polynomial }\end{array}$ & \multicolumn{2}{|c|}{$\begin{array}{c}\text { squared } \\
\text { free } \\
\text { distance }\end{array}$} & \multicolumn{3}{c|}{$\begin{array}{c}\text { asymptotic } \\
\text { codinggain } \\
\text { over M/2- } \\
\text { AM or PRS }\end{array}$} \\
\cline { 2 - 8 } & \multicolumn{1}{|c|}{$\mathrm{H}^{0}$} & $\mathrm{H}^{1}$ & TCM & TCPRS & 4 AM & $8 \mathrm{AM}$ & $16 \mathrm{AM}$ \\
\hline 2 & 5 & 2 & 9 & 10 & 0 & 0.8 & 1.0 \\
3 & 13 & 4 & 10 & 10 & 0 & 0.8 & 1.0 \\
4 & 23 & 4 & 11 & 12 & 0.8 & 1.6 & 1.8 \\
5 & 45 & 10 & 13 & 14 & 1.5 & 2.2 & 2.4 \\
6 & 103 & 24 & 14 & 14 & 1.5 & 2.2 & 2.4 \\
7 & 235 & 126 & 16 & 16 & 2.0 & 2.8 & 3.0 \\
8 & 515 & 362 & 16 & 18 & -- & 3.5 & 3.5 \\
8 & 515 & 362 & 17 & 16 & 2.6 & - & -- \\
\hline
\end{tabular}

(a)

\begin{tabular}{|r|r|r|r|c|c|c|c|}
\hline$V$ & \multicolumn{2}{|c|}{$\begin{array}{c}\text { encoder } \\
\text { check } \\
\text { polynomial }\end{array}$} & \multicolumn{2}{c|}{$\begin{array}{c}\text { square } \\
\text { free } \\
\text { distance }\end{array}$} & \multicolumn{2}{c|}{$\begin{array}{c}\text { asymptotic } \\
\text { coginggain } \\
\text { over M/2- } \\
\text { QAM }\end{array}$} \\
\cline { 2 - 8 } & \multicolumn{1}{|c|}{$\mathrm{H}^{0}$} & \multicolumn{1}{|c|}{$\mathrm{H}^{1}$} & \multicolumn{1}{|c|}{$\mathrm{H}^{2}$} & TCM & TCPRS & 16QAM & 64 QAM \\
\hline 2 & 7 & 2 & 0 & 4 & 6 & 1.8 & 1.8 \\
3 & 11 & 2 & 4 & 5 & 6 & 1.8 & 1.8 \\
4 & 23 & 4 & 16 & 6 & 6 & 1.8 & 1.8 \\
5 & 41 & 6 & 10 & 6 & 6 & 1.8 & 1.8 \\
6 & 101 & 016 & 064 & 7 & 8 & 3.0 & 3.0 \\
7 & 203 & 014 & 042 & 8 & 8 & 3.0 & 3.0 \\
8 & 401 & 056 & 304 & 8 & 8 & 3.0 & 3.0 \\
9 & 1001 & 0346 & 0510 & 8 & 8 & 4.0 & 4.0 \\
\hline
\end{tabular}

(b)

Tabe 1. Free distance and asymptotic coding gain of (a) one- and (b) two-dimensional TCPRS.

\section{Performance at moderate SNR}

The error coefficient of TCPRS is relatively small such that at moderate SNR its performance is relatively good, as we shall see in this section. The event error probability of TCPRS is bounded by the union bound 


$$
A_{d_{\text {free }}} Q\left(\sqrt{d_{\text {free }}^{2} / 2 N_{0}}\right) \leq \operatorname{Pr}(e) \leq \sum_{d=d_{\text {free }}}^{\infty} A_{d} Q\left(\sqrt{d^{2} / 2 N_{0}}\right)
$$

where $d^{2}$ represents the squared Euclidean distance between signal sequences, $A_{d}$ is the average multiplicity of codeword at distance $d^{2}$ from a specific codeword where the average is taken over all codewords in the code, $d_{f r e e}^{2}$ is the squared free distance and $N_{0}$ is the single-sided PSD of Gaussian noise. Using the inequality $Q(\beta) \leq \frac{\exp \left(-\beta^{2} / 2\right)}{\sqrt{2 \pi} \beta}$, the upper bound becomes

$$
\operatorname{Pr}(e) \leq \sqrt{\frac{N_{0}}{\pi d_{\text {free }}^{2}}} \sum_{d=d_{\text {free }}}^{\infty} A_{d} \exp \left(-d^{2} / 4 N_{0}\right) .
$$

The general algorithm for calculating the free distance and error probability of a irregular trellis based on Equation 18 is proposed by Divsalar [11]. A computer program is written in $\mathrm{C}$ to run on a MASSCOMP 6450 workstation. Recursive numerical algorithm for manipulating sparse matrix is employed to make the computation of large matrix possible. The squared free distance predicted by Theorem 2 and listed in Table 1 is verified by the program for short and moderate constraint length. To illustrate the performance of TCPRS at moderate SNR, the error coefficients $A_{d_{f r e e}}$ of 8-AM TCM and the corresponding TCPRS are listed in Table 2(a) for constraint length up to 5. The 16-QAM TCM and the corresponding TCPRS are also listed in Table 2(b). There are two points to be noted in Table 2. First, comparing the error coefficient of TCM and TCPRS, the latter is usually an order of magnitude smaller, and sometimes two orders of magnitude smaller, such that the degradation in receiver sensitivity is not as bad as $3 \mathrm{~dB}$. Secondly, although the squared free distance of TCPRS can remain the same for several different constraint lengths, e.g., in the 16-QAM TCPRS it remain 6 for constraint length 2,3 , and 4 , the error coefficient does decrease as the constraint length increases, and thus give a modest increase in coding gain. As a rule of thumb[13], a decrease of 16 times in error coefficient amounts to a coding gain of $1 \mathrm{~dB}$ at median SNR. In Figure 3 we plot the upper bound of event error probablity of 8-AM TCM and the corresponding TCPRS for constraint 4 and 5 . The performance of TCPRS with respect to TCM is only $1 \mathrm{~dB}$ at error rate $10^{-6}$, and is even smaller at lower SNR.

\section{Conclusion}

The code search and performance analysis for TCPRS has been presented in this paper. Good TCPRS codes can be found among good TCM codes. As compared to TCM codes, this class of TCPRS codes found in this paper features a degradation of 2 to $2.5 \mathrm{~dB}$ at extremely high SNR, and a degradation of only $1 \mathrm{~dB}$ at median SNR, while the spectral behavior is improved.

\begin{tabular}{|c|l|l|}
\hline \multirow{2}{*}{$v$} & \multicolumn{2}{|c|}{ error coefficients of 8-AM } \\
\cline { 2 - 3 } & TCM & TCPRS \\
\hline 2 & 1.25 & 0.0660 \\
3 & 0.633 & 0.0198 \\
4 & 0.613 & 0.00628 \\
5 & 0.460 & 0.00953 \\
\hline
\end{tabular}

(a)

\begin{tabular}{|c|l|l|}
\hline \multirow{2}{*}{$v$} & \multicolumn{2}{|c|}{ error coefficients of 16 -QAM } \\
\cline { 2 - 3 } & TCM & TCPRS \\
\hline & & \\
2 & 0.5 & 0.423 \\
3 & 1.28 & 0.0185 \\
4 & 2.37 & 0.00466 \\
\hline
\end{tabular}

(b)

Table 2. Error coefficients of TCM and TCPRS for (a) 8-AM and (b) 16-QAM constellations.

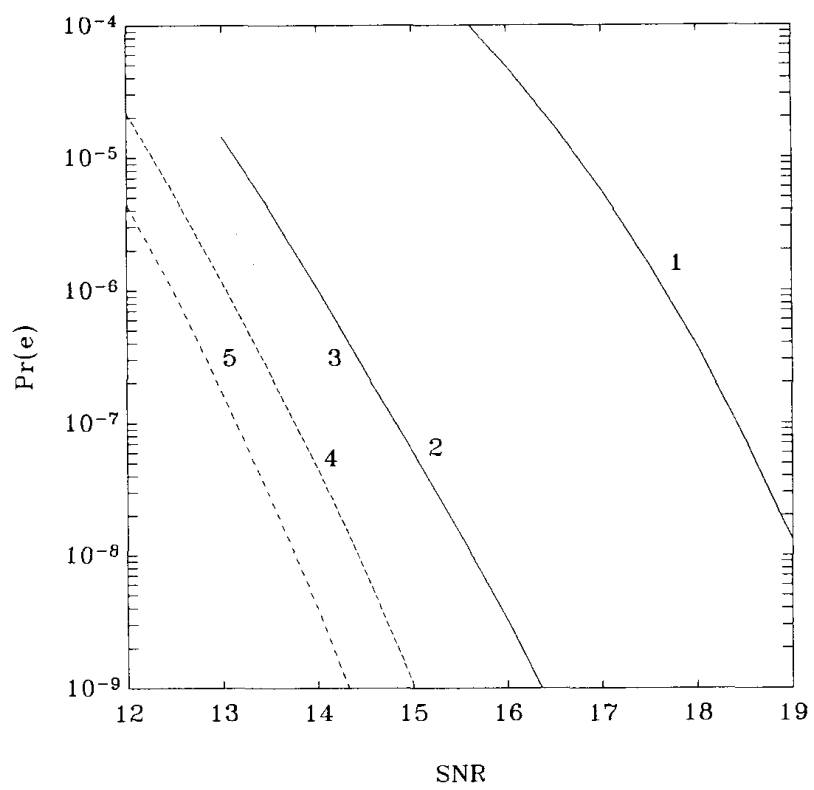

Figure 2. Event error probability of TCPRS and TCM curve 1: uncoded 4-AM, 2: constraint length 4 8-AM TCPRS, 3: constraint 58-AM TCPRS, 4: constraint length 4 8-AM TCM, 5: constraint 5 8-AM TCM 


\section{References}

[1] P. Kabal and S. Pasupathy, "Partial-response signaling," IEEE Trans. Commun., vol. COM-23, no. 9, pp. 921-943, Sept. 1975.

[2] S. Pasupathy, "Correlative coding," IEEE Commun. Mag., vol. 13, no. 7, pp. 4-11, July 1977.

[3] C.-E. Sundberg, "Continuous phase modulation," IEEE Commun. Mag., vol. 24, no. 4, pp. 25-36, Apr. 1986.

[4] G. D. Forney, Jr., "Maximum-likelihood sequence estimation of digital sequences in the presence of intersymbol interference," IEEE Trans. Inform. Theory, vol. IT-18, no. 3, pp. 363-378, May 1972.

[5] G. Ungerboeck, "Channel coding with multilevel/phase signals," IEEE Trans. Inform. Theory, vol. IT-28, no. 1, pp. 55-67, Jan. 1982.

[6] J. K. Wolf and G. Ungerboeck, "Trellis coding for partial-response channels," IEEE Trans. Commun., vol. COM-34, no. 8, pp. 765-773, Aug. 1986.

[7] A. R. Calderbank, C. Heegard, and T. A. Lee, "Binary convolutional codes with application to magnetic recording," IEEE Trans. Inform. Theory, vol. IT-32, no. 6 , pp. 797-815, Nọv. 1986.

[8] J. W. Ketchum, "Performance of trellis codes for Mary partial response," Conf. Proc. Globecom'87, Tokyo, Japan, pp. 1727-1724, 1987.

[9] G. D. Forney, Jr. and A. R. Calderbank, "Coset codes for partial response channels; or, coset codes with spectral nulls," IEEE Trans. Inform. Theory, vol. IT-35, no. 5, pp. 925-943, Sept. 1989.

[10] M. Rouanne and D. J. Costello, "An algorithm for computing the distance spectrum of trellis codes," IEEE $J$. Select. Areas Commun., vol. SAC-7, no. 6, pp. 929-940, Aug. 1989.

[11] D. Divsalar, M. K. Simon and J. H. Yuen, "Trellis coding with asymmetric modulations," IEEE Trans. Commun., vol. COM-35, no. 2, pp. 130-141, Feb. 1987.

[12] P. R. Chevillat and E. Eleftheriou, "Decoding of trelliscoded signals in the presence of intersymbol interference and noise," IEEE Trans. Commun., vol. COM-37, no. 7 , pp. 669-676, July 1989 .

[13] G. D. Forney, Jr., "Coset codes-Part I: Introduction and geometrical classification," IEEE Trans. Inform. Theory, vol. IT-34, no. 5 pp. 1123-1151, Sep. 1988. 